\title{
Editorial: Smart Energy Systems
}

\author{
Agostino Gambarotta $^{1 *}$, Konstantinos Kyprianidis ${ }^{2}$ and \\ Panayotis Dimopoulos Eggenschwiler ${ }^{3}$
}

${ }^{1}$ Center for Energy and Environment-CIDEA, University of Parma, Parma, Italy, ${ }^{2}$ School of Business Society and Engineering, Division of Sustainable Energy Systems, Mälardalen University, Västerås, Sweden, ${ }^{3} E M P A-S w i s s$ Federal Laboratories for Materials Science and Technology, Swiss Federal Laboratories for Materials Science and Technology, Dübendorf, Switzerland

Keywords: smart \& intelligent technologies, energy, energy consumption, energy storage, energy networks integration, renewable resources, modeling and simulation

\section{Editorial on the Research Topic}

\section{Smart Energy Systems}

In recent years the field of Energy has had to meet increasing challenges involving several issues, from primary resources consumption, to costs, pollutant emissions, resilience and accessibility. One of the main effects has been a substantial change in the Energy scientific and technical paradigms -based on typical centralised architectures, with large power grids designed and managed relying on empirical rules derived from the experience of operators- towards more intricate realities.

As a consequence, the use of terms such as "Smart Energy" and "Smart Energy Systems" are becoming nowadays very common involving a broader approach. While "Smart Grids" focus primarily on the electricity sector, "Smart Energy Systems" address an integrated and holistic approach that includes all involved Energy sectors (electricity, heating, cooling, industry, buildings, mobility, etc.) seeking for more effective and efficient solutions for the Sustainable Energy Transition.

As a matter of fact, the complexity of Energy Systems and Networks has been growing in the last decade and it is apparent that this trend will continue. The rapid diffusion of Renewable Energy Sources (RES), the development of new technologies (from energy generation and use, to IoT and IoE-based facilities), the requirements of the Energy markets (costs, emissions, flexibility, resilience) are posing severe challenges. The fluctuating availability of RES (solar and wind) and users' demands (households, mobility, etc.) are pushing the need for Energy Storage Systems, which nowadays can count on several options such as Thermal Energy Storage, Batteries, Compressed Air Energy Storage, Power-to-Gas and Power-to-X plants, etc.

However, from an overarching point of view all these considerations outline new and very promising scenarios for the next future. Multi-Energy and District-Energy Systems paradigms have started to attract the interest of both private and public institutions, since they appear as promising solutions to lower cost and environmental impact of the energy production, distribution and use. The key element for their success is incorporating Distributed Energy Generation as well as Sector integration, i.e., integration of Energy Networks (electricity, heating and cooling, gas) to take advantage of all available synergies and to allow for a further increase in the share of RES. Enhancements in energy generation, distribution, storage and use can be optimally integrated and managed involving digital/ICT technologies to allow for the shift to modern energy infrastructures, which should be secure, resilient and reliable.

Yet, the pace at which these technologies are being adopted should be at the same pace the innovation growth, and this involves tackling both scientific and technical issues. Within this context this special collection on "Smart Energy Systems" intends to provide contributions that highlight solutions, methodologies, approaches, and tools towards optimal design, management, and control for conversion, distribution, storage and use of energy. 
The papers collected in this Special Issue offer insights in the complex field of Smart Energy Systems. They cover a broad range of topics (as it should be expected, considering the amplitude of the subject) involving both the improvement of performance of defined components of generation systems, and the optimisation of the performance of complex networks where specific technologies are integrated.

Internal Combustion Engines will most likely continue to play an interesting role -not only in the Energy transition phase- in heavy-duty and stationary applications (e.g., as a back-up for RES generation) even fuelled with sustainable fuels (as biomass or syngas from Power-to-Gas plants). Lean-burn gas/dual-fuel combustion is an interesting topic in this direction, and development of suitable injectors to improve the ignition processes is investigated in Süess et al. using an optically accessible test rig to develop a novel injector design.

For the same purposes Micro-Gas Turbines can be considered for combined heat and power generation plants, which have to be properly integrated in the existing Energy Networks. The study developed by Aslanidou et al. is specifically focused on the optimisation of a fleet of Micro-Gas Turbines for domestic applications in Smart Energy Systems. In the paper a physicsbased model and a data-driven model with machine learning capabilities have been combined for predicting system behavior, with particular attention to diagnostic tasks. The framework has been implemented on a fleet of Micro-Gas Turbines for optimal operation and production planning to fit them in the future green energy system.

With reference to the heating sector (which accounts for $40 \%$ of the Energy consumption in Europe), Smart District Heating Networks can play a key role in the reduction of Energy consumption and related emissions. The exploitation of bidirectional Energy exchange between District Heating Networks and connected users is investigated by Ancona et al. analysing the possibility of including a "prosumer" (i.e., a customer who can both consume and produce heat) in an existing small/medium District Heating Network. Using a specific simulation tool, this study shows how the position of a prosumer in the network and the related thermal power affect the performance of the network, giving indications to optimise its architecture and management.
Energy Storage is a fundamental issue to overcome the problem of strongly fluctuating RES and therefore cannot be disregarded in Smart Energy Systems. In the paper by Tiano et al. an energy storage system consisting of under water compressed air energy storage (UWCAES) is presented with reference to a case study where RES (wind and photovoltaic) are considered. Specific tools are used to model and optimise size and operation of both the energy production and the storage system as well as its auxiliary services.

The papers collected in this Special Issue are an example on how the design of Systems and Networks for the sustainable Energy transition requires a holistic approach that allows to join the research on specific components with the optimisation of the architecture and management strategies of the networks where they are integrated. For this reason, this Special Issue should be considered as a first collection on the topic of Smart Energy Systems and Networks.

\section{AUTHOR CONTRIBUTIONS}

All authors listed have made a substantial, direct, and intellectual contribution to the work. AG wrote the first draft, KK and PDE carefully revised the article. All authors approved the final version.

Conflict of Interest: The authors declare that the research was conducted in the absence of any commercial or financial relationships that could be construed as a potential conflict of interest.

Publisher's Note: All claims expressed in this article are solely those of the authors and do not necessarily represent those of their affiliated organizations, or those of the publisher, the editors and the reviewers. Any product that may be evaluated in this article, or claim that may be made by its manufacturer, is not guaranteed or endorsed by the publisher.

Copyright $\odot 2022$ Gambarotta, Kyprianidis and Dimopoulos Eggenschwiler. This is an open-access article distributed under the terms of the Creative Commons Attribution License (CC BY). The use, distribution or reproduction in other forums is permitted, provided the original author(s) and the copyright owner(s) are credited and that the original publication in this journal is cited, in accordance with accepted academic practice. No use, distribution or reproduction is permitted which does not comply with these terms. 